Original Article

\title{
Effect of arm swaying from wearing a self-applied arm sling with a loop on walking and other mobility skills
}

\author{
Shiori Kakae, RPT, MS ${ }^{1}$, AkiYoshi TAKami, RPT, PhD ${ }^{1) *}$, Misato Makino, RPT, PhD ${ }^{1)}$ \\ HideKI YoshIDA, RPT, $\mathrm{PhD}^{1)}$ \\ 1) Nursing Home Asakuraen: 18-32 Shimorokujocho, Fukui-shi, Fukui 918-8135, Japan
}

\begin{abstract}
Purpose] This study aimed to investigate how wearing a self-applied arm sling with a loop during rising, standing up, and walking affects the magnitude of arm swaying and activities among elderly individuals compared with wearing a triangular bandage. [Participants and Methods] Fourteen elderly individuals participated in the study. The methods involved attaching a triaxial accelerometer to an arm wearing a triangular bandage or arm sling and conducting a 5-m walk test, sit-to-stand test five times, and rising up. We then calculated the time required for each action, and the acceleration, vibration intensity, and coefficient of variation of the affected arm; these were then compared between the two groups. [Results] All the participants were able to put on the arm sling with a loop. The step rate with a triangular bandage was higher than that without one. Swaying in the front/back direction in standing up was greater with the triangular bandage. [Conclusion] The self-applied arm sling with a loop was shown to have less front/back swaying during standing up. Wearing a triangular bandage may have narrowed the stride and increased the step rate due to discomfort. This result should be applied with caution, because it remains unclear whether arm slings with loops are advantageous.

Key words: Arm sling, Walk, Sit to stand
\end{abstract}

(This article was submitted May 20, 2020, and was accepted Jul. 9, 2020)

\section{INTRODUCTION}

Arm slings and triangular bandages have long been worn as a means to handle shoulder subluxation caused by stroke. They have also been shown to be effective at mitigating subluxation and alleviating worsening pain ${ }^{1)}$. An effect on walking when they are worn by hemiplegic stroke patients has also been reported; wearing an arm sling reportedly has a positive effect on walking speed, the load factor on the affected side, the double support phase, stride, and step width ${ }^{2,3}$.

Triangular bandages, meanwhile, have an advantage in that when worn, they make it possible to smoothly shift the center of gravity to the unaffected side, but it has been noted that attempting to correct for this produces a compensatory action when the center of gravity is displaced to attempted side, and the pelvic inclination angle increases, and it tries to hold the posture in sitting posture ${ }^{4}$. The greatest disadvantage in clinical practice is that they cannot be put on without assistance. Long-term usage to wear everyday life is also often found to result in misalignment of the triangular bandage. In other words, an arm sling with loop, because the problem and fixability of the mounting of the triangle width is weak, there is a possibility to compensate for the fact that the negative effect on the operation.

When we therefore used a force plate to analyze walking, the triangular bandage was found to have a stronger outward lateral force component than wearing an arm sling, leading to the presumption that irregular arm movements would affect the $\operatorname{legs}^{5}$. No document that can be found, however, has involved actually measuring swaying of the paralyzed-side arm while

*Corresponding author. Akiyoshi Takami (E-mail: a-takami@hirosaki-u.ac.jp)

(C)2020 The Society of Physical Therapy Science. Published by IPEC Inc.

(c) (i) $\odot$ This is an open-access article distributed under the terms of the Creative Commons Attribution Non-Commercial No Deriva-

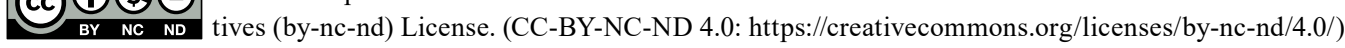


wearing a triangular bandage or arm sling.

The purpose of the present study is therefore to use a triaxial accelerometer to study how wearing an arm sling with loop that a person can put on without assistance affects arm swaying as compared to when a triangular bandage is worn, as well as differences in required times for actions, when elderly individuals close to the age of frequent onset of stroke rise up, stand up, and walk, for the purpose of basic data collection before use for strokes.

\section{PARTICIPANTS AND METHODS}

Participants were 15 users (6 males and 9 females; ages $85.9 \pm 5.6$ years) of day service center A who understood the main purpose of the task and gave written consent. Exclusion criteria covered those presenting with apparent dementia, those with severe higher brain dysfunction, those with severe sensory impairment, those with severe parkinsonism, and those with orthopedic disorders of the spine and lower limbs that interfere with walking, standing, sitting, or rising up.

In terms of ethical procedures, this task was approved by the Hirosaki University Hospital School of Health Sciences Ethics Committee (approval number: 2018-019).

Methods involved first conducting a preliminary survey to investigate the rate of wearing triangular bandage or arm sling with loop, described below, by putting it on without assistance, as well as the time required to do so. The other items on the preliminary survey were sex, age, prior history (orthopedic disease, history of surgery), whether or not a T-cane is used, and the Mini Mental State Examination (MMSE).

The measurement device was a triaxial accelerometer (MicroStones MVP-RF10-AC, a small 10-channel wireless motion recorder). The sampling frequency was $200 \mathrm{~Hz}$. The triaxial accelerometer was fitted to the arm wearing the triangular bandage or arm sling. For the 5-m walk test and the five times sit-to-stand test in the following measurement items, it was fitted to the midpoint between the radial styloid process and the ulnar styloid process. For the action of rising up, it was attached to the most anterior surface in the sagittal plane of the acromion of the side wearing the triangular bandage or arm sling.

Acceleration and vibration intensity were measured with the triaxial accelerometer for each of the actions. Acceleration was analyzed by dividing the maximum amplitude into the $\mathrm{X}$-axis (left/right), the Y-axis (up/down), and the Z-axis (front/ back), and the composite value $\mathrm{r}=\sqrt{ }(\mathrm{x} 2+\mathrm{y} 2+\mathrm{z} 2)$ was also calculated $\left.{ }^{6}\right)$. The coefficient of variation of acceleration, to represent swaying, was also calculated in the same manner.

The 5-m walk test involved walking $5 \mathrm{~m}$ at a comfortable pace, with a 3-m buffer before and after for a total of $11 \mathrm{~m}$. In terms of walking aids, only use of a T-cane was accepted, in consideration of the report from Yeon-Gyu et $\mathrm{al}^{7)}$. The time required to walk $5 \mathrm{~m}$ was measured, as was the number of steps, to calculate the walking speed and step rate. The acceleration, coefficient of variation, and vibration intensity were measured with the triaxial accelerometer. Measurements were taken twice under each of the conditions, and the mean value was calculated.

The five times sit-to-stand test involved setting up a 40-cm-high chair with arm rests and measuring the time required to stand up from sitting 5 consecutive times. The acceleration, coefficient of variation, and vibration intensity were measured with the triaxial accelerometer. Measurements were taken twice under each of the conditions, and the mean value was calculated.

For the action of rising up, the time required to sit up at the edge of the bed from the supine position on the bed was measured. The participant was understood to be sitting at the edge of the bed at the time when an examiner was able to determine visually that the ischium on both sides was loaded evenly. The acceleration, coefficient of variation, and vibration intensity were measured with the triaxial accelerometer. Rising up is often an action that involves turning around, and there are complicated three-dimensional changes in direction, so only a composite of vectors was employed. Measurements were taken twice under each of the conditions, and the mean value was calculated.

Measurement conditions were: a) non-wearing condition; normal walking, with no restrictions on the arms. b) Arm sling with loop; Walking while wearing an arm sling with loop on the right arm. c) Triangular bandage; walking while wearing a triangular bandage on the right arm. These 3 conditions were implemented in random order, spaced out by at least 1 day in between each. The arm sling with loop has a loop $(26-30 \mathrm{~cm})$ formed so that the forearm can be placed in it, at both ends of a sling measuring $7 \mathrm{~cm}$ wide and about 120-130 long; the material is polyester rubber and is elastic (Fig. 1a). The triangular bandage used is widely available and made of cotton, measuring $105 \mathrm{~cm} \times 105 \mathrm{~cm} \times 150 \mathrm{~cm}$ (Fig. 1b).

All statistical processing involved performing a normality check beforehand (Shapiro-Wilk), with a multiple comparison test (Tukey's test) of walking speed, step rate, five times sit-to-stand time, and rising up time with respect to the 3 conditions. A paired t-test was run between the 2 groups wearing the triangular bandage or arm sling with loop, from the data obtained with the triaxial accelerometer. SPSS for Windows Ver. $22.0 \mathrm{~J}$ (IBM) was used for statistical analysis, with the level of significance set to $\mathrm{p}<0.05$.

\section{RESULTS}

The basic attributes shall be described. The 5-m walk test and the five times sit-to-stand test were performed on 14 elderly participants who use the day service center A. There were 13 participants for only the action of rising up (1 participant dropped out midway due to low back pain) (Table 1). 


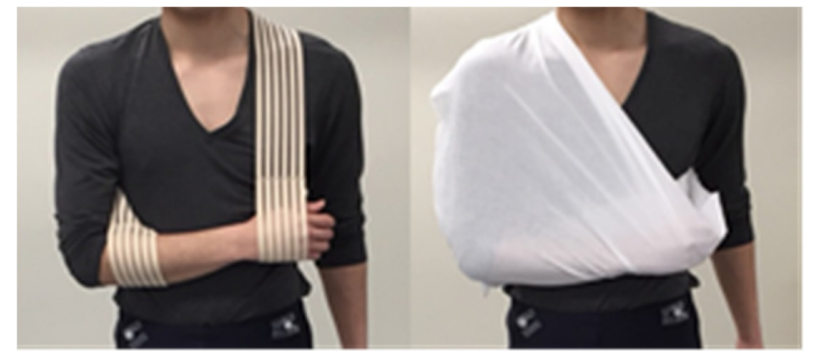

Table 1. Participant characteristics $(n=14)$

\begin{tabular}{lc}
\hline Gender (males/females) & $5 / 9$ \\
Age (years) & $85.9 \pm 5.8(77-95)$ \\
Handedness (right/left) & $13 / 1$ \\
Use of a T-cane (yes/no) & $6 / 8$ \\
MMSE (points) & $23.8 \pm 4.8(16-30)$ \\
\hline Mean \pm SD (min-max). &
\end{tabular}

Fig. 1. Wearing an arm sling with loop (left) and wearing a triangular bandage (right)

In terms of the rate of being able to put on the arm sling with loop or triangular bandage without assistance, all participants were able to with the arm sling with loop. The mean time required was 42.13 seconds. None of the participants were capable with the triangular bandage.

Table 2 shows the results for walking speeds and step rates, acceleration, coefficient of variation, and vibration intensity in the 5-m walk test. No significant differences were found in the 3 conditions. Walking speed did not show a significant difference between the 3 conditions. Results showed the step rate to be significantly higher when wearing the triangular bandage than not wearing it.

Table 3 shows the results for times, acceleration, coefficient of variation, and vibration intensity in the five times sit-tostand test. The Z-axis (front/back) and composite of acceleration were found to be significantly greater when the triangular bandage is worn than when the arm sling with loop is worn. The coefficient of variation and vibration intensity did not exhibit significant differences in the 3 conditions.

Table 4 shows the results for times, acceleration, coefficient of variation, and vibration intensity in the action of rising up. No significant differences were found between the 3 conditions for the action of rising up.

\section{DISCUSSION}

The present task investigated how wearing or not wearing an arm sling with loop or a triangular bandage affects walking speed and arm swinging in the 5-m walk test. Results showed that wearing the triangular bandage resulted in a significantly greater step rate than not wearing it. Walking speed did not exhibit any significant difference between the 3 conditionswearing the arm sling with loop, wearing the triangular bandage, and not wearing either. This suggests that stride may be smaller only for the triangular bandage. Walking under non-specific conditions such as walking backwards is handled by decreasing stride and increasing the step rate ${ }^{8)}$. In other words, wearing a triangular bandage appears to have created greater discomfort, similar to walking on uneven terrain or unstable walking, compared to not wearing one. Shuit et al. ${ }^{9)}$ stated that a torso-immobilizing sling did not affect step rate compared to not wearing one among stroke patients. In the present study, there was no significant difference between wearing the arm sling with loop and not wearing one. In other words, wearing the arm sling with loop appears not to have created any more discomfort than not wearing one. Makino et al. ${ }^{10)}$ used a threedimensional analysis device and force plate to analyze walking with an arm sling with loop of the same type as in the present study, finding a slight difference in pelvic rotation as compared to not wearing one, but reported that there were essentially no kinematic changes, raising the possibility that an arm sling with loop poses no kinematic difference from not wearing one.

Arm acceleration during walking showed no significant difference between the 2 conditions. Yavuzer et al. ${ }^{2)}$ reported that wearing an arm sling improved walking speed and walking parameters in stroke patients, but did not produce changes in healthy individuals. The elderly participants of the present study yielded similar results, being close to relatively healthy individuals. Though right/left swaying has been considerable in previous research, there was no tendency for major left/right arm swaying, suggesting that at comfortable walking speeds for elderly individuals, there is not very much arm swaying during use.

The effect on the five times sit-to-stand test was investigated with regard to duration of the five times sit-to-stand test and with regard to arm swaying, by whether or not an arm sling with loop or a triangular bandage was being worn. The results showed no significant difference in duration of the five times sit-to-stand test between the 3 conditions. The elderly participants of the present study usually walk independently or use a T-cane, and may have been able to largely maintain their leg muscle strength. The use of a chair with armrests, done for safety, in the present test also resulted in some individuals using the armrests to stand up from sitting, resulting in no effect on the time to execute the action between the 3 conditions.

There was significantly greater arm acceleration, when a triangular bandage was worn, in the Z-axis (front/back) and the composite. The X-axis (left/right) and Y-axis (up/down) of acceleration, the coefficient of variation, and the vibration intensity showed no significant differences between the 3 conditions. The significantly large Z-axis (front/back) when wearing a triangular bandage shows that the arm wearing it shakes forward/backward during rising from sitting. In other words, 
Table 2. Result of walking speed, step rate, acceleration, coefficient of variation, and vibration intensity in $5 \mathrm{~m}$ walk test $(\mathrm{n}=14)$

\begin{tabular}{lccc}
\hline & Non-wearing condition & Arm sling with loop & Triangular bandage \\
\hline Walking speed (m/min) & $47.90 \pm 15.27$ & $49.18 \pm 18.11$ & $49.90 \pm 17.45$ \\
Step rate (steps/min) & $116.13 \pm 31.05$ & $127.75 \pm 42.31$ & $142.34 \pm 49.72^{*}$ \\
\hline Acceleration (m/sec ${ }^{2}$ ) & & & \\
X-axis (left/right) & $5.00 \pm 1.37$ & $5.32 \pm 1.11$ \\
Y-axis (up/down) & $6.33 \pm 2.28$ & $7.75 \pm 4.13$ \\
Z-axis (front/back) & $6.34 \pm 2.10$ & $5.98 \pm 2.63$ \\
Composite & $9.13 \pm 2.32$ & $9.86 \pm 4.33$ \\
\hline Coefficient of variation & & \\
X-axis (left/right) & $0.56 \pm 2.17$ & $0.50 \pm 0.24$ \\
Y-axis (up/down) & $0.63 \pm 0.17$ & $0.57 \pm 0.26$ \\
Z-axis (front/back) & $0.42 \pm 0.24$ & $0.55 \pm 0.24$ \\
Composite & $0.29 \pm 0.14$ & $0.27 \pm 0.12$ \\
\hline Vibration intensity & $5.34 \pm 2.08$ & $5.79 \pm 3.27$ \\
\hline
\end{tabular}

Mean \pm SD. ${ }^{*} \mathrm{p}<0.05$ only non-wearing condition vs. triangular bandage.

Table 3. Result of the times, acceleration, coefficient of variation, and vibration intensity for the five times sit-to-stand test $(\mathrm{n}=14)$

\begin{tabular}{|c|c|c|c|}
\hline & Non-wearing condition & Arm sling with loop & Triangular bandage \\
\hline Time (sec) & $15.74 \pm 6.49$ & $15.91 \pm 5.97$ & $15.14 \pm 5.56$ \\
\hline \multicolumn{4}{|l|}{ Acceleration $\left(\mathrm{m} / \mathrm{sec}^{2}\right)$} \\
\hline $\mathrm{X}$-axis (left/right) & & $5.83 \pm 3.16$ & $5.19 \pm 1.59$ \\
\hline Y-axis (up/down) & & $9.64 \pm 4.28$ & $10.87 \pm 5.36$ \\
\hline Z-axis (front/back) & & $8.39 \pm 3.23$ & $10.38 \pm 3.46^{*}$ \\
\hline Composite & & $13.20 \pm 4.62$ & $15.17 \pm 4.84^{*}$ \\
\hline \multicolumn{4}{|l|}{ Coefficient of variation } \\
\hline $\mathrm{X}$-axis (left/right) & & $0.61 \pm 0.24$ & $0.55 \pm 0.21$ \\
\hline Y-axis (up/down) & & $0.68 \pm 0.21$ & $0.59 \pm 0.28$ \\
\hline Z-axis (front/back) & & $0.47 \pm 0.18$ & $0.50 \pm 0.20$ \\
\hline Composite & & $0.38 \pm 0.12$ & $0.36 \pm 0.13$ \\
\hline Vibration intensity & & $5.91 \pm 1.91$ & $7.30 \pm 3.19$ \\
\hline
\end{tabular}

Mean \pm SD. $* \mathrm{p}<0.05$.

Table 4. Result of the times, acceleration, coefficient of variation, and vibration intensity for the action of rising up $(n=13)$

\begin{tabular}{lccc}
\hline & Non-wearing condition & Arm sling with loop & Triangular bandage \\
\hline Time $(\mathrm{sec})$ & $4.92 \pm 1.99$ & $4.38 \pm 1.57$ & $4.52 \pm 1.47$ \\
\hline $\begin{array}{c}\text { Acceleration }\left(\mathrm{m} / \mathrm{sec}^{2}\right) \\
\quad \text { Composite }\end{array}$ & $16.17 \pm 2.23$ & $15.79 \pm 2.16$ & 0.284 \\
\hline $\begin{array}{l}\text { Coefficient of variation } \\
\text { Composite }\end{array}$ & $0.30 \pm 0.98$ & $0.27 \pm 0.98$ & 0.248 \\
\hline Vibration intensity & $11.38 \pm 1.79$ & $11.54 \pm 1.36$ & 0.577 \\
\hline
\end{tabular}

Mean \pm SD.

the arm wearing it separates from the torso during rising from sitting. When performing trunk forward tilt motion during standing operation and sitting operation, the upper limbs wearing a triangle width, away from the trunk, it is conceivable that is shaking front/back. This may be a hindrance when one is executing the action of rising from sitting. Contrary to expecta- 
tions, wearing the arm sling with loop had higher immobilization to the torso than the triangular bandage. Han et al. ${ }^{11)}$ have noted that the energy cost for putting on a sling is low for stroke patients, and the disadvantage of arm immobilization may potentially also affect the energy cost.

As for the effect on the action of rising up, there was no significant difference between the 3 categories in terms of the duration of the action of rising up. The elderly participants of the present study have maintained their torso muscle strength, resulting in no effect on whether or not they used the contralateral arm from the side on which they rose up.

Arm acceleration showed no significant difference between the 2 conditions. The 2 conditions are approximate values, and the triaxial accelerometer was attached to the acromion, but is not thought to have affected the magnitude of arm swaying from wearing. As already noted, the elderly participants of the present study had maintained their torso and leg muscle strength, and possibly were not using the contralateral arm from the side for rising up, leaving acceleration unaffected.

In conclusion, the arm sling with loop is made of a highly elastic material so that it can be put on without assistance. Accordingly, it has a disadvantage in terms of immobilization as compared to a triangular bandage. Swaying of the arms themselves, however, was largely unchanged, and the arm sling with loop was actually shown to have less front/back swaying in standing up and the like, as the triangular bandage causes the arms to be separated from the body. It is also possible that the triangular bandage created discomfort during walking by wearing. Thus, since the arm sling with loop can be put on without assistance and, when worn, creates less discomfort, it presumably has the potential to be useful.

In terms of limitations of the present study, participants were not stroke patients themselves but rather elderly individuals; for patients experiencing hemiplegia or with subluxation, the bone head needs to be tugged into the glenoid cavity. In general, this result should be applied with caution, and does not make it possible to assert that the arm sling with loop is at an advantage. In the future, we will study in stroke patients with shoulder subluxation, and we would like to conduct further research on the usefulness of the arm sling with loop.

\section{Conflict of interest}

There are no conflicts of interest to disclose.

\section{REFERENCES}

1) Brooke MM, de Lateur BJ, Diana-Rigby GC, et al.: Shoulder subluxation in hemiplegia: effects of three different supports. Arch Phys Med Rehabil, 1991, 72: 582-586. [Medline]

2) Yavuzer G, Ergin S: Effect of an arm sling on gait pattern in patients with hemiplegia. Arch Phys Med Rehabil, 2002, 83: 960-963. [Medline] [CrossRef]

3) Hwang YI, An DH: Immediate effects of an elastic arm sling on walking patterns of chronic stroke patients. J Phys Ther Sci, 2015, 27: 35-37. [Medline] [CrossRef]

4) Sawada Y, Abe T, Asamura N, et al.: Effect of an armsling on posture:Sitting in healthy subjects and patients with hemiplegia. Jpn Soc Private Med Coll Phys Ther Res, 2015, 21: 36-39 (in Japanese).

5) Kakae S, Takami A, Makino M, et al.: Effects of wearing a fixed trunk arm sling on walking and balance in healthy subjects. Journal of Fukui Physical Therapy, 2018, 22: 11-16 (in Japanese).

6) Matsumoto Y, Takai S, Shiota H, et al.: Study on the construction of the tremor analysis and evaluation system using 3-axis accelerometers. Nagaoka University of Technology Research Report, 2002, 24: 99-106 (in Japanese).

7) Jeong YG, Jeong YJ, Koo JW: The effect of an arm sling used for shoulder support on gait efficiency in hemiplegic patients with stroke using walking aids. Eur J Phys Rehabil Med, 2017, 53: 410-415. [Medline]

8) Takami A, Wakayama S: Characteristics of backward walking in acute stroke patient—comparison of forward walking with backward walking. Tohoku Rigaku Ryouhougaku, 2009, 21: 104-110 (in Japanese).

9) Shuit C, Takami A, Makino M, et al.: The relationship between arm swing and walking abilities in hemiplegia patients. The Hirosaki Medical Journal, 2019, 69: 119-123.

10) Makino M, Takami A, Kakae S, et al.: Effects of an arm sling on the trunk and pelvis while walking. Clin Gait Anal Forum Jpn, 2019, 6: 1-7 (in Japanese).

11) Han SH, Kim T, Jang SH, et al.: The effect of an arm sling on energy consumption while walking in hemiplegic patients: a randomized comparison. Clin Rehabil, 2011, 25: 36-42. [Medline] [CrossRef] 